\title{
A comparison of simple correction and functional feedback in schema learning
}

\author{
ROBERT A. SMALLWOOD and MALCOLM D. ARNOULT \\ Texas Christian University, Fort Worth, Texas 76129
}

\begin{abstract}
The role of knowledge of results (KR) in a schematic concept formation task was clarified by manipulating the specificity of information provided during feedback. Ss learned to discriminate between pairs of random forms from two schema families. Verbal correction was compared with a functional KR procedure in which the schema prototypes were superimposed over test stimuli. In no case did verbal correction improve learning in comparison with functional KR. Differences in acquisition persisted in a retention test.
\end{abstract}

The development of the ability to categorize stimuli without external sources of information or prior familiarization with the relevant schema has been termed schematic concept formation (Evans, 1967). Studies have demonstrated that schematic concept formation can occur without extrinsic feedback or knowledge of results (KR) (Evans \& Arnoult, 1967; Evans \& Edmonds, 1966; Rosser, 1967). Other studies, including those by Tracy and Evans (1967) and Brown, Walker, and Evans (1968), have indicated further that the ability to learn to categorize visual patterns is not appreciably facilitated by KR in the form of simple "right-wrong" correction.

As a plausible explanation for the occurrence of schematic concept formation without extrinsic feedback, Edmonds, Mueller, and Evans (1966) have suggested that information in the stimulus patterns themselves is sufficient to allow selection of relevant attributes in a fashion analogous to cluster analysis. This interpretation appears to be in accord with the Gibsons' (1955) proposition that Ss can learn to differentiate higher order variables without extrinsic KR.

Less easily explained is the striking fact that when correction is administered, little or no facilitation has been observed. While numerous authors (e.g., Miller, 1966; Edmonds \& Mueller, 1967; Tracy, 1971) have offered hypotheses to account for the lack of facilitative effects of correction, the explanation initially proposed by Breckenridge, Rankin, and Wright (1969) seems most tenable; that is, on tasks in which there is a moderate level of stimulus redundancy, learning is possible on the basis of intrinsic information alone. Intrinsic information, however, is less than perfectly reliable. Extrinsic feedback in the form of correction, in conjunction with the less than perfectly reliable intrinsic information, may confuse $S$ and make it difficult for him to determine accurately why he is correct or incorrect. As a consequence, his performance level is not greater than, and may even be less than, he could achieve on the basis of intrinsic information alone. The learning of appropriate attributes assumed necessary to make perceptual discriminations in a schematic concept formation task may therefore be unimproved by the administration of KR in the form of correction.

The purpose of the present study was to clarify the role of $K R$ as it is administered in a schematic concept formation task. It was hypothesized that the administration of $\mathrm{KR}$ in conjunction with moderately redundant stimuli would not confuse $S$ if the feedback was made more informative. Since $S$ is presumably required to learn the nature of the prototype and the range of permissible variation of the members of the schema class, KR which directly provides that information should be more effective than a simple correction procedure. Superimposing outline drawings of the prototypes over the test stimuli directly provides that information. This method for providing KR was designated as functional knowledge of results (FKR) and was provided at several different levels of specificity. It was hypothesized that schema learning would be directly related to the specificity of this functional KR and that FKR, in general, would be more effective than verbal correction or no KR. It was further hypothesized that differences observed during acquisition would persist in a test of retention of schema learning.

\section{METHOD}

\section{Subjects}

The Ss were 224 undergraduates enrolled in an introductory psychology course at Texas Christian University. Each $S$ was given class credit for participating in the experiment.

\section{Apparatus}

Two programmable Carousel projectors were used to project slides on a white screen approximately $8-10 \mathrm{ft}$ in front of the Ss, who were run in groups of up to seven at a time. In front of each $S$ was a response box with two keys marked "S" and "D." Stimulus presentation, response collection, and all timing were controlled by electromechanical programming equipment.

\section{Stimuli}

Patterns were computer-generated about two prototypes to form schematic clusters, as described in detail elsewhere (Aiken $\&$ Brown, 1971). The 70 pairs of random forms selected from two classes were divided into seven groups of 10 , which were matched for judged similarity as a control for discrimination difficulty. In each group there were an equal number of "same class" and "different class" pairs. 
Table 1

Knowledge of Results Conditions Employed for Each Group in Each Phase of the Task

\begin{tabular}{|c|c|c|c|c|c|}
\hline \multirow[b]{2}{*}{ Group } & \multicolumn{2}{|c|}{$\begin{array}{l}\text { Training Phase } \\
\text { (10 Trials) }\end{array}$} & \multicolumn{2}{|c|}{$\begin{array}{l}\text { Acquisition Phase } \\
\text { (40 Trials) }\end{array}$} & \multirow{2}{*}{$\begin{array}{c}\begin{array}{c}\text { Retention Phase } \\
\text { (30 Trials) }\end{array} \\
\text { KR }\end{array}$} \\
\hline & FKR & VKR & FKR & VKR & \\
\hline $\mathrm{AB}$ & $\begin{array}{l}\text { Relevant Prototype } \\
\text { Tree } 1 \text { and Tree } 2\end{array}$ & No & $\begin{array}{l}\text { Relevant Prototype } \\
\text { A and B }\end{array}$ & No & None \\
\hline$A B+V$ & $\begin{array}{l}\text { Relevant Prototype } \\
\text { Tree } 1 \text { and Tree } 2\end{array}$ & Yes & $\begin{array}{l}\text { Relevant Prototype } \\
\mathrm{A} \text { and } \mathrm{B}\end{array}$ & Yes & None \\
\hline $\begin{array}{l}A+V \\
B+V \\
N \\
N+V \\
\text { VKR } \\
\text { NKR }\end{array}$ & $\begin{array}{l}\text { Prototype Tree } 1 \\
\text { Prototype Tree } 2 \\
\text { Neutral Tree } \\
\text { Neutral Tree } \\
\text { None } \\
\text { None }\end{array}$ & $\begin{array}{l}\text { Yes } \\
\text { Yes } \\
\text { No } \\
\text { Yes } \\
\text { Yes } \\
\text { No }\end{array}$ & $\begin{array}{l}\text { Prototype A } \\
\text { Prototype B } \\
\text { Neutral Prototype* } \\
\text { Neutral Prototype* } \\
\text { None } \\
\text { None }\end{array}$ & $\begin{array}{l}\text { Yes } \\
\text { Yes } \\
\text { No } \\
\text { Yes } \\
\text { Yes } \\
\text { No }\end{array}$ & $\begin{array}{l}\text { None } \\
\text { None } \\
\text { None } \\
\text { None } \\
\text { None } \\
\text { None }\end{array}$ \\
\hline
\end{tabular}

*Octagon

\section{Training}

A major difficulty in same-different classification tasks is communicating to $S$ that his response should be in terms of the class membership of the individual stimuli rather than in terms of the identity or nonidentity of the particular visual forms being shown. For this purpose, a training task was developed in which two classes of trees were randomly constructed, with mem bers deviating from their respective prototypes according to a set of rules which established boundaries for the length and width of the foliage and the trunk of each tree, with a different set of rules pertaining to each of the two classes of trees. For Class 1 trees, the ratio of height to width for foliage was $<5 / 3$ and for the trunk was $>2 / 1$, while for Class 2 trees, the height-width ratio for foliage was $>1 / 2$ and for the trunk was $<5 / 2$. The Ss were shown line drawings of pairs of the trees and asked to judge whether they belonged to the same "species" or to different "species" of trees. For each S, 10 such trials were provided, with KR conditions essentially identical to those administered during the experiment itself; for example, FKR consisted of superimposing prototype trees over test stimuli. The various KR conditions will be described below in further detail.
Following the training trials, the Ss were questioned to determine their understanding of the task requirements.

\section{Acquisition}

Each $S$ received four blocks of 10 trials in which each stimulus pair was presented for a period of $8 \mathrm{sec}$, during which $S$ pressed a button to indicate whether he judged a pair to have been drawn from the "same" class or from "different" classes of forms. This interval was followed by a 5 -sec period during which KR was provided while the stimuli remained in view.

The eight experimental groups were distinguished by the kind of KR provided during training and acquisition, as shown in Table 1.

Group $A B$. For these Ss, functional knowledge of results was provided by superimposing outline drawings of the prototype forms over the stimulus forms, as illustrated in Fig. 1a. In every case, the prototype was actually the prototype of the schema class from which that stimulus had been drawn.

Group $A B+V$. This group received the same kind of FKR and also received verbal correction (VKR) by means of an intercom.
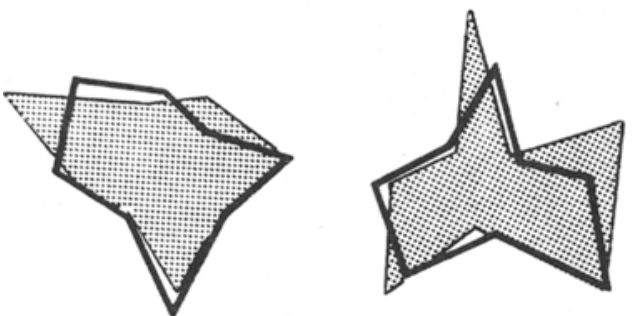

a

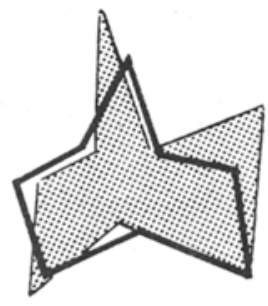

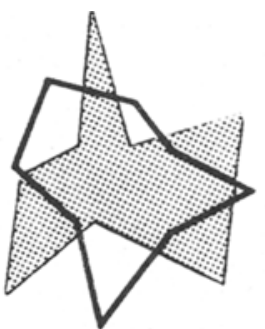



C

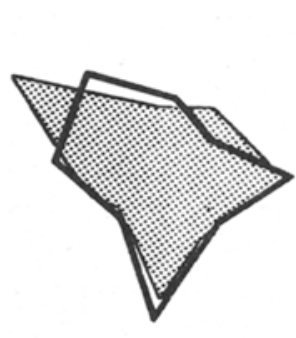

6
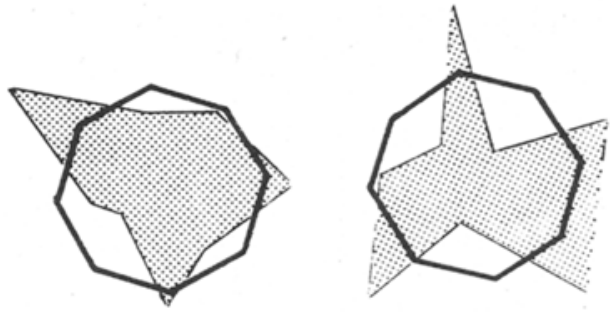

Fig. 1. Examples of functional KR conditions showing (a) actual prototype forms, (b) Schema Class A forms, (c) Scherna Class B forms, and (d) neutral octagon forms superimposed over test stimuli. 
The Ss were told whether they should have responded "similar" or "different."

Group $A+V$. For this group, the prototype from Schema Class A was superimposed over both figures on every trial, as shown in $F$ ig. 16 . In addition, VKR was provided.

Group $B+V$. This group was treated like the previous group, except that the prototype of Schema Class B was superimposed over the trial stimuli, as illustrated in Fig. 1c. For both of these groups, VKR was always provided because a pilot study had shown that for this experimental condition Ss were unable to understand the nature of the task without the aid of VKR.

Group N. Because of the way in which the stimuli were originally constructed, a regular octagon was a form that was equally prototypical of both schema classes. An octagon was used, therefore, as a neutral prototype and superimposed over both stimuli, as shown in Fig. 1d.

Group $N+V$. The octagon was used for FKR and supplemented with VKR.

Group VKR. This group received VKR only.

Group NKR. This group received no KR of any kind.

\section{Retention}

After completing 40 acquisition trials, all Ss were given 30 retention trials, during which no KR was provided to any of the groups. All groups, except NKR, were given brief additional instructions explaining the change in procedure. Group NKR received a $3-\mathrm{min}$ rest period between acquisition and retention phases for the purpose of equating the time interval between phases for all groups.

\section{RESULTS}

\section{Acquisition Phase}

Figure 2 graphically represents the mean number of correct classifications (maximum $=10$ ) as a function of blocks of trials for each of the KR conditions. A two-way analysis of variance, with one between-group factor (KR conditions) and one within-group factor (blocks of trials) was performed, with total correct classifications as the dependent variable. There was a significant $K R$ main effect $[F(7,216)=8.98, p<.01]$ and a significant trials main effect $[F(3,648)=27.21$, $\mathrm{p}<.01]$, while interaction among groups over trials was not significant. A Newman-Keuls procedure testing differences among ordered means across trials showed that groups performed significantly better on Blocks II, III, and IV than on Block I $(\mathrm{p}<.01)$. The Newman-Keuls procedure applied to the KR conditions showed: (a) that performance by Groups $A B, A B+V$, and $B+V$ was significantly better than performance by the remaining groups, although they did not differ among themselves; (b) that Group A+V performed significantly better than Groups $\mathrm{N}, \mathrm{N}+\mathrm{V}$, VKR, and NKR, and (c) that the overall performance by Group $N$ was superior to that of Groups N+V, VKR, and NKR, with no significant differences in performance among these last three.

Thus, during the acquisition phase, several outcomes were noteworthy. First, all groups demonstrated some learning regardless of whether they received FKR, VKR, or no KR. Second, in no case did a group receiving VKR perform significantly better than its corresponding group which did not receive VKR. Third, the group exposed to only Prototype B performed as well as the groups whose

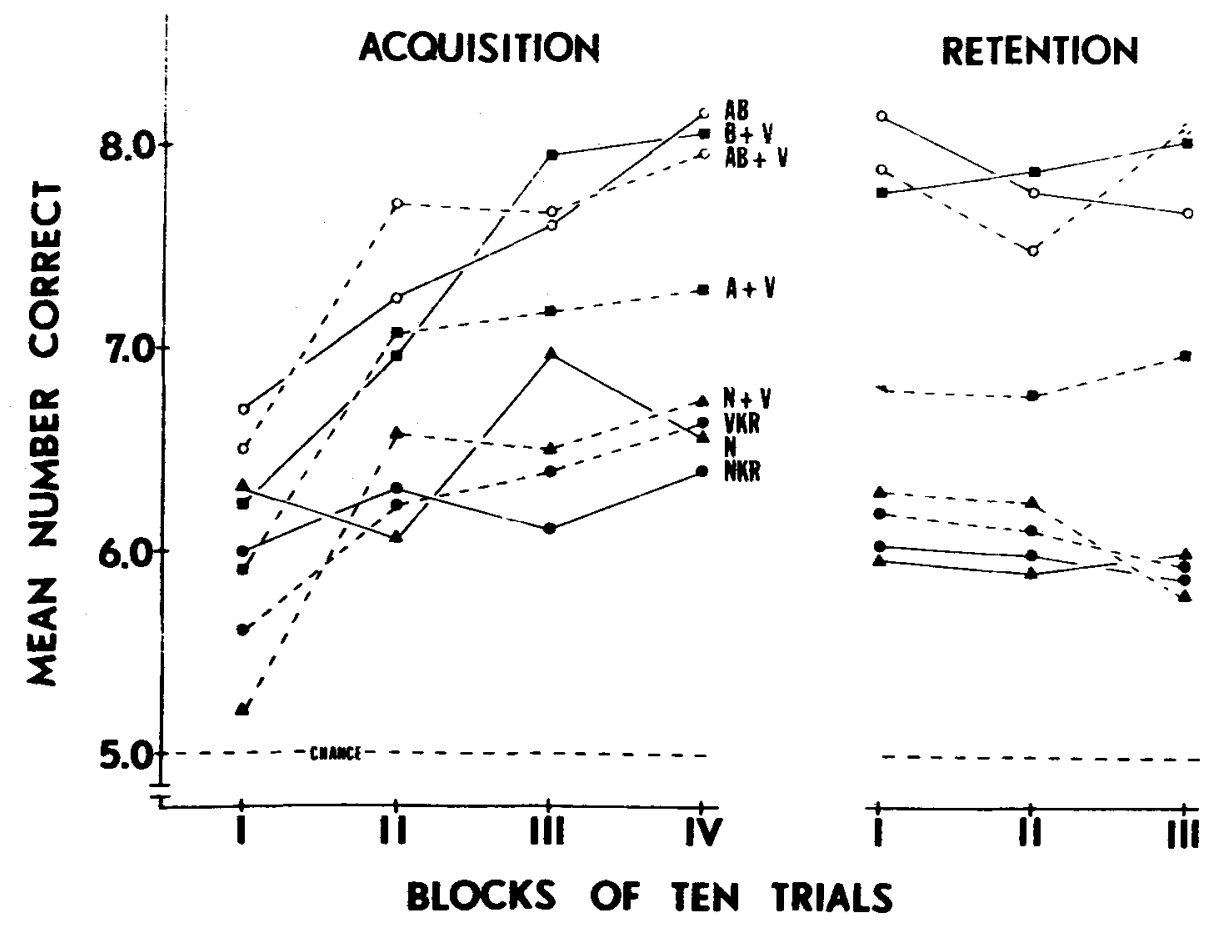

Fig. 2. Mean number of correct classifications as a function of blocks of 10 trials during acquisition and retention. 
FKR involved both prototypes, whereas the group exposed only to Prototype A made significantly fewer correct classifications. Finally, although the overall performance of Group $\mathrm{N}$ was significantly better than that of the remaining groups, it should be noted that on the last block of acquisition trials the groups receiving the neutral prototype as FKR were performing no differently from the groups which received no FKR at all. This last result indicates that the FKR procedure, per se, did not importantly influence the acquisition of schema learning. It neither facilitated nor retarded learning in comparison with the standard procedures not involving FKR. When the FKR procedure did include relevant information, however, schema learning was enhanced.

\section{Retention Phase}

Performance in the retention phase was again scored in terms of total correct classification responses as a function of blocks of trials, and is graphically represented in Fig. 2. A two-way analysis of variance was performed which indicated significant between-group main effects $[F(7,216)=1532, p<.01]$. No other significant effects were obtained in this analysis. The Newman-Keuls procedure applied to the retention data showed that the overall performance of Groups $A B$, $A B+V$, and $B+V$ was superior to that of Group $A+V$, which, in turn, was superior to that of the remaining groups, which did not differ from each other.

Some of the groups showed a significant decrement in performance between Block IV of acquisition and Block I of retention $[F(1,216)=5.76, p<.01]$. The Newman-Keuls procedure indicated significant decrements $(p<.01)$ for the lowest five groups, but not for the three groups $(A B, A B+V$, and $B+V)$ which had performed best during acquisition. Finally, no group showed significant change from Block I to Block III of the retention test.

\section{DISCUSSION}

Taken as a whole, the results of the experiment support the position that the learning of perceptual classes is based on a schema-plus-correction principle, and that such learning can be strongly facilitated by procedures designed to help the learner to identify the prototype and to recognize the ways in which class members deviate from it. The results also support previous research showing that a verbal correction procedure does not significantly facilitate this kind of learning. Presumably, any effective correction procedure must permit the learner to recognize the nature of his error. On tasks where learning is possible on the basis of less than perfectly reliable intrinsic stimulus information, extrinsic feedback in the form of verbal correction may be ineffective in facilitating performance. On the other hand, when feedback is characterized by functionally relevant information, schema learning progresses in a fashion typical of traditional human learning tasks.

It is not the case, therefore, that providing knowledge of results is an ineffective procedure in schema learning. Rather, the results show that simple correction procedures are ineffective in this kind of task. The procedure designated as functional KR provides $S$ with the kind of information needed in order to improve his performance, and its effectiveness is clearly related to the degree to which the information provided in the feedback is specific to the task requirements.

Although the results were generally in accord with the experimental hypotheses, mention should be made of two unusual outcomes. First, in the acquisition phase, it is not clear why Group $B+V$ performed as well as the two $\mathrm{AB}$ groups and better than Group A+V. Detailed analysis failed to reveal that any particular stimulus pairs were notably more or less difficult for one of these groups than for the other. Possibly the result is related to particular characteristics of the prototype forms. Perhaps, if a prototype is particularly distinctive or quite high in association value, Ss are able to discriminate by classifying each stimulus either as "a member of Class B" or as "not a member of Class B." If the random procedures used in constructing the stimuli had produced different prototypes, it is possible that these two groups would not have differed in performance.

Second, it is not clear why Groups $A B, A B+V$, and $B+V$ showed no performance decrement in moving to the retention task while the remaining groups did. Since various KR conditions were represented in these three groups, it is difficult to find a common factor other than their generally high level of performance to account for this finding. Equally surprising is the significant decrement shown by the NKR group, since for that group the retention phase involved no change in procedure whatsoever.

Finally, it should be noted that the rapid improvement in performance of some of the experimental groups contrasts sharply with the much slower rates of learning demonstrated both in this experiment and in previous research when either no KR or only simple correction is provided. It is possible, therefore, that insofar as the natural environment provides feedback of the sort described here as "functional," the original learning of perceptual classes may occur much faster than the results of previous research on this problem would suggest.

\section{REFERENCES}

Aiken, L. S., \& Brown, D. R. A feature utilization analysis of the perception of pattern class structure. Perception \& Psychophy sics, 1971, 9, 279-283.

Attneave, F. Transfer of experience with a class-schema to identification-learning of patterns and shapes. Journal of Experimental Psychology, 1957, 54, 81-88.

Breckenridge, R. L., Rankin, W. C., \& Wright, A. D. The effect of $K R$ on ratings of pattern similarity. Psychonomic Science, $1969,15,223-224$.

Brown, B. R., Walker, D. W., \& Evans, S. H. Schematic concept 
formation as a function of constraint redundancy and knowledge of results. Psychonomic Science, 1968, 11, 75-76.

Edmonds, F. M.. \& Mueller, M. R. Effects of incidental training and reinforcement on mixed schema learning. Psychonomic Science, 1966, 10, 75-76.

Edmonds, F. M., Mueller, M. R., \& Evans, S. H. Effects of knowledge of results on mixed schemata discrimination. Psychonomic Science, 1966,6,377-378.

Evans. S. H. A brief statement of schema theory. Psychonomic Science, 1967, 8, 87-88.

Evans, S. H., \& Arnoult, M. D. Schematic concept formation: Demonstration in a free sorting task. Psychonomic Science, $1967,9,221-222$.

Evans, S. H., \& Edmonds, E. M. Schema discrimination as a function of training. Psychonomic Science, $1966,5,303-304$.

Gibson, J. J., \& Gibson, E. J. Perceptual learning: Differentiation or enrichment? Psychological Review, 1955, 62, 32-41.
Miller, L. B. Learning a visual pattern discrimination with and without KOR. Psychonom ic Science, 1966, 6, 199-200.

Rosser, E. M. Categorization and discrimination of tone sequences. Unpublished doctoral dissertation, Harvard University, April 1967.

Tracy, J. F. The effects of types of supplem entary information, example deviation, redundancy and experience on categorization in a four category sorting task. Unpublished doctoral dissertation. Texas Christian University, 1971.

Tracy, J. F., \& Evans, S. H. Supplementary information in schem atic concept formation. Psychonomic Science, 1967, 9 , 313-314.

(Received for publication October 17, 1973 ; revision received February $7,1974$. 